\title{
The Effect of Spatial Location of Fermentation Pit on Prokaryotic Community Diversity in Pit Mud for Chinese Strong-Flavor Baijiu Production
}

\section{Xiaolong $\mathrm{Hu}$}

Zhengzhou University of Light Industry

\section{Dahong Feng}

Zhengzhou University of Light Industry

\section{Miao Yu}

Zhengzhou University of Light Industry

\section{Sifan Wang}

Zhengzhou University of Light Industry

\section{Yong Zhang}

Zhengzhou University of Light Industry

\section{Lei Chi}

Zhengzhou University of Light Industry

\section{Zhigang Zhang}

Jiahu Distillery Group Co., Ltd

\section{Peixin He}

Zhengzhou University of Light Industry

\section{Yongliang Wang}

Jiahu Distillery Group Co., Ltd

Wenyou Zhu ( $\square$ Zhuwenyou1105@163.com )

Yibin University

\section{Research Article}

Keywords: fermentation pit mud, spatial heterogeneity, microbial community, physicochemical factors, Chinese strong flavor Baijiu

Posted Date: June 30th, 2021

DOI: https://doi.org/10.21203/rs.3.rs-653850/v1

License: (c) (1) This work is licensed under a Creative Commons Attribution 4.0 International License.

Read Full License 

1 The effect of spatial location of fermentation pit on prokaryotic community

2 diversity in pit mud for Chinese strong-flavor Baijiu production

3

4 Xiaolong $\mathrm{Hu}^{1,2}$, Dahong Feng ${ }^{2}$, Miao $\mathrm{Yu}^{2}$, Sifan $\mathrm{Wang}^{2}$, Yong Zhang ${ }^{2}$, Lei Chi ${ }^{2}$, Zhigang Zhang ${ }^{3}$,

5 Peixin $\mathrm{He}^{2}$, Yongliang Wang ${ }^{3}$, Wenyou $\mathrm{Zhu}^{1 *}$

6

7 1.Faculty of Agriculture, Forestry and Food Engineering, Solid-state Fermentation resource

8 Utilization Key Laboratory of Sichuan Province, Yibin University, Yibin 644000, China

9 2.School of Food and Bioengineering, Collaborative Innovation Center of Production and Safety,

10 Zhengzhou University of Light Industry, Zhengzhou 450000, China

11 3. Jiahu Distillery Group Co., Ltd., Luohe 462400, China

12

13

\section{Correspondence}

14 Wenyou Zhu, Yibin University, No. 8, Jiusheng Road, East Section of Wuliangye Avenue, Yibin

15 City, Sichuan Province, China. Email: Zhuwenyou1105@163.com; 


\section{Background}

Chinese strong-flavor baijiu (CSFB) accounts for more than $70 \%$ of all Chinese liquor markets. Diverse microbes in pit mud found in the fermentation pit play a key role in CSFB production. However, the effect of spatial location on the diversity and structure of the microbial community in pit mud is still poorly understood

\section{Results}

Prokaryotic microbes in different pit mud (4- and 40-year) were analyzed by using Illumina MiSeq sequencing of 16S rRNA gene. The samples were collected from pit mud that was 4 and 40 years old in the top, middle, and under parts of the cellar walls and at the bottom of them. The results showed there was no significant difference ( $>0.05$ ) in the physicochemical factors, the Observed OTU, and $\alpha$-diversity between the pit mud of two different ages. The index of the 4-year-old pit mud was lower (except for total acid, which was higher than) than that of the 40-year-old pit mud. The $\mathrm{pH}$, total acid, Observed OTU, Chao 1, ACE in the 4-year-old pit mud (in the top and middle part of the cellar wall) and the 40-year-old-pit mud(in the top part of the cellar wall) had significant $(\mathrm{p}<0.05)$ difference. At the phylum level, Firmicutes (56.70\%) and Bacteroidetes (26.56\%) accounted for more than $80 \%$ of the overall level. For the 4-year-old pit mud sample, the dominant genuses at each location were Proteiniphilum, Lactobacillus, and Caproiciproducens, while for the 40-year-old pit mud, there were 10 common genuses accounted for more than $50 \%$ of the dominant genuses in different pit mud belong to Clostridia. The RDA analysis results showed that $85.71 \%$ of the dominant bacteria positively correlated with $\mathrm{pH}$, and especially the 
most correlated with the total acid and available phosphorus, so its content was significantly related to the dominant bacteria $(\mathrm{p}<0.01)$. Therefore, it inferred that the total acid and available phosphorus were the main physicochemical factors that affected the spatial distribution of prokaryotic microbial communities in the pit mud of cellars from the liquor distillery.

\section{Conclusions}

Comparing the young pit mud and the old pit mud, the structure and physicochemical factors of the prokaryotic microbial community in pit mud from Henan liquor-making company changed, and the changes occurred in the spatial location of different pits.

Clostridia, which accounted for more than $50 \%$ of all types of pit mud, were responsible for the effect of total acid and available phosphorus on the microbial community. Based on the above, it provides a theoretical basis for Henan liquormaking companies in maintaining pit mud.

Keywords: fermentation pit mud; spatial heterogeneity; microbial community; physicochemical factors; Chinese strong-flavor Baijiu. 


\section{Background}

Chinese strong-flavor baijiu (CSFB) is one of the four flavors of liquor in China. Under the joint action of the environment, Daqu, and microorganisms in the cellar,

Chinese strong-flavor baijiu was obtained through a series of complex substances and energy metabolism[1] of bacteria. With the acclimation and development of pit mud microorganisms, a unique microbial community has gradually formed in a few years or hundreds of years, and the unique cellar aroma of CSFB depends on the unique microbial community within these pit mud[2]. Besides, there were differences in the living environment of microorganisms in different locations of the pit pool, and typical spatial heterogeneity existed in the pit mud microbial community. These differences affected the production and metabolism of microorganisms in the pit mud [3]. Therefore, it is necessary to study the cellar mud of different ages and in different spatial locations, and use a systematic method to analyze the distribution characteristics of the microbial community in pit mud.

In recent years, with the use of pyrosequencing technology, Tao et al. [6] analyzed the structures of prokaryotic microbial communities in various ages $(1,10,25$, and 50 years) of pit mud, and found the cellar mud needed at least 25 years to reach maturity; Using metagenomic sequencing, Guo et al. [17]found the microorganisms

Euryarchaeota and Bacteroidetes in cellar mud increased in 50, 140, 220, and 440 years as the age of the cellar increased; Liu et al. [7] first reported the Caproiciproducens for Chinese Baijiu production in pit mud by using Illumina MiSeq sequencing technology; $\mathrm{Li}[4]$ and $\mathrm{Hu}[5]$ and others concluded that the composition of pit mud microbial community was significantly affected by environmental variables. At present, there are many studies on pit mud from distilleries in Sichuan, Guizhou, and other provinces[1, 2,3,5,6,7], most of them are about the analysis of the diversity 
of pit mud microbial community in different pit ages, qualities, and geographical locations, few related studies on the distribution characteristics of the microbial community of CSFB mud from distilleries in Henan province. Zhang Huimin et al. [8] found there was a certain difference in the composition of microbial community between the young and the old cellar mud. This shows that there is a correlation between the distribution and the composition of the microbial community in pit mud. Therefore, in this study, we collected samples from a 4- and 40-year-old pit mud occupied on the top, middle, under, and bottom cellar wall in a CSFB company in Henan. Illumina MiSeq sequencing technology was used to analyze the distribution characteristics of the cellar mud microbial community. It provided plentiful information for the study of the microecology of pit mud in Henan liquor distilleries and also provided a theoretical basis for the specific influence of different ages and spatial locations of pit mud in its distribution of microbial communities.

\section{Results}

\section{Physicochemical factors of different pit mud}

In Table 1, compared with the 40-year pit mud, the water content, $\mathrm{pH}$ value, ammonium nitrogen, and available phosphorus content of the 4-year-old pit mud were lower but its total acid content was significantly higher $(\mathrm{p}<0.05)$. There was a significant difference $(\mathrm{p}>0.05)$ neither in the moisture content of different aged pit mud nor in the physicochemical factors of 40-year-old pit mud in different locations. In comparison with the 40-year-old pit mud, the $\mathrm{pH}$ value of the 4-year-old pit mud was lower $(\mathrm{p}<0.05)$ (on the top and middle cellar wall) but the same at the bottom while its total acid content at all locations was higher $(\mathrm{p}<0.05)$. Furthermore, the 

contents of NH4+ and AP were the lowest in the top 4-year-old pit mud. In summary,

103 the physicochemical factors of the top 4-year-old pit mud were different from those of 104 the under 4-year-old pit mud and the 40-year-old pit mud. 
Table 1. Physicochemical factors, number of OTUs of prokaryotic microbial communities, and $\alpha$-diversity in pit mud from different pit

\begin{tabular}{|c|c|c|c|c|c|c|c|c|}
\hline \multirow{2}{*}{ Variable } & \multicolumn{4}{|c|}{ 4-year } & \multicolumn{4}{|c|}{ 40-year } \\
\hline & $4 \mathrm{~T}$ & $4 \mathrm{M}$ & $4 U$ & $4 \mathrm{~B}$ & $40 \mathrm{~T}$ & $40 \mathrm{M}$ & $40 \mathrm{U}$ & $40 \mathrm{~B}$ \\
\hline Moisture (\%) & $37.1 \pm 1.92 \mathrm{a}$ & $40.1 \pm 1.34 \mathrm{a}$ & $40.32 \pm 1.67 \mathrm{a}$ & $37.8 \pm 0.66 \mathrm{a}$ & $37.49 \pm 6.33 \mathrm{a}$ & $41.93 \pm 4.22 \mathrm{a}$ & $44.54 \pm 3.28 \mathrm{a}$ & $38.63 \pm 0.93 a$ \\
\hline $\mathrm{pH}$ & $4.17 \pm 0.07 \mathrm{a}$ & $4.09 \pm 0.29 \mathrm{a}$ & $5.27 \pm 0.92 \mathrm{ab}$ & $6.33 \pm 2.65 \mathrm{abc}$ & $8.64 \pm 0.07 \mathrm{c}$ & $8.44 \pm 0.28 \mathrm{c}$ & $7.26 \pm 1.17 \mathrm{bc}$ & $8.26 \pm 0.22 \mathrm{c}$ \\
\hline Total acid $\left(m g \cdot g^{-1}\right)$ & $10.86 \pm 0.66 \mathrm{c}$ & $9 \pm 0.09 c$ & $3.41 \pm 2.19 b$ & $2.17 \pm 1.76 \mathrm{ab}$ & $0.31 \pm 0 \mathrm{a}$ & $0.31 \pm 0.22 \mathrm{a}$ & $1.32 \pm 0.55 \mathrm{ab}$ & $0.31 \pm 0 \mathrm{a}$ \\
\hline $\mathrm{NH} 4+\left(\mathrm{mg} \bullet 100 \mathrm{~g}^{-1}\right)$ & $38.45 \pm 5.98 \mathrm{a}$ & $46.85 \pm 16.39 \mathrm{ab}$ & $136.43 \pm 40.24 \mathrm{abc}$ & $206.99 \pm 110.44 \mathrm{c}$ & $171.56 \pm 49.38 b c$ & $186.79 \pm 35.37 \mathrm{c}$ & $173.91 \pm 43.34 \mathrm{bc}$ & $116.44 \pm 42.91 \mathrm{abc}$ \\
\hline $\mathrm{AP}\left(\mathrm{mg} \cdot 100 \mathrm{~g}^{-1}\right)$ & $1.84 \pm 0.25 \mathrm{a}$ & $5 \pm 2.87 \mathrm{a}$ & $11.01 \pm 1.72 \mathrm{ab}$ & $14.09 \pm 8.36 \mathrm{ab}$ & $12.89 \pm 12.87 \mathrm{ab}$ & $21.21 \pm 15.38 \mathrm{ab}$ & $27.9 \pm 4.65 b$ & $13.69 \pm 10.75 \mathrm{ab}$ \\
\hline OTU & $392 \pm 9.9 \mathrm{a}$ & $405.5 \pm 62.93 a$ & $706 \pm 182.43 \mathrm{ab}$ & $663 \pm 156.98 \mathrm{ab}$ & $1079.5 \pm 434.87 \mathrm{bc}$ & $1242.5 \pm 200.11 \mathrm{c}$ & $1148 \pm 111.72 b c$ & $1065 \pm 93.34 b c$ \\
\hline Chao 1 & $431.19 \pm 5.46 a$ & $551.75 \pm 131.88 \mathrm{a}$ & $846.89 \pm 191.28 \mathrm{ab}$ & $832.89 \pm 69.62 \mathrm{ab}$ & $1310.61 \pm 568.21 b c$ & $1500.39 \pm 236.49 \mathrm{c}$ & $1430.29 \pm 148.98 b c$ & $1368.53 \pm 71.08 \mathrm{bc}$ \\
\hline ACE & $436.54 \pm 7.56 \mathrm{a}$ & $527.27 \pm 83.37 \mathrm{a}$ & $867.98 \pm 213.43 \mathrm{ab}$ & $855.1 \pm 100.04 \mathrm{ab}$ & $1357.09 \pm 620.61 b c$ & $1560.85 \pm 239.45 \mathrm{c}$ & $1487.28 \pm 155.43 \mathrm{bc}$ & $1415.26 \pm 77.1 \mathrm{bc}$ \\
\hline Shannon & $3.72 \pm 0.39 \mathrm{ab}$ & $3.22 \pm 0.52 \mathrm{a}$ & $3.93 \pm 0.19 \mathrm{ab}$ & $4.03 \pm 0.07 \mathrm{ab}$ & $4.24 \pm 0.48 b$ & $4.66 \pm 0.27 b$ & $4.09 \pm 0.62 \mathrm{ab}$ & $4.12 \pm 0.31 \mathrm{ab}$ \\
\hline Simpson & $0.94 \pm 0.03 \mathrm{a}$ & $0.85 \pm 0.12 \mathrm{a}$ & $0.95 \pm 0 \mathrm{a}$ & $0.96 \pm 0 \mathrm{a}$ & $0.93 \pm 0.04 \mathrm{a}$ & $0.97 \pm 0.01 \mathrm{a}$ & $0.93 \pm 0.05 \mathrm{a}$ & $0.95 \pm 0.02 \mathrm{a}$ \\
\hline
\end{tabular}


108

109

110

111

112

As shown in Table 1, the number of OTUs each sample obtained, based on $97 \%$ similarity in $16 \mathrm{~S}$ rRNA gene sequences, ranged from 392 to 1242 . Chao 1 index was between 431.19 and 1500.39. The Shannon index was between 3.22 and 4.66. From the analysis of pit age, both the number of OTUs and the alpha diversity index of the 4-year-old pit mud were lower than those of the 40-year-old pit mud. And the OTU, Chao 1, and ACE in the top 4-year-old pit mud were significantly $(\mathrm{p}<0.05)$ lower than those in the top 40-year-old pit mud. The Shannon index of the middle 4-year-old pit mud was significantly lower $(\mathrm{p}<0.05)$ than that in the top 40-year-old pit mud. It inferred that the diversity of microbial species was low in 4-year-old pit mud. With the deepening of the pit mud, there was no significant difference in alpha diversity between the 4 -and the 40 -year-old pit mud at different locations, but certain differences in the change law existed. In the alpha diversity index, the 4-year-old pit mud at the top location was lower while the 40 -year-old pit mud at the top location was higher than that at the under location.

\section{Beta diversity of microbial communities in different pit muds}

Clustering of pit mud samples based on UniFrac analysis

The cluster analysis of species UniFrac (with species and its abundance taken into account) showed that the samples were classified into two categories(Fig. 1). The under and bottom locations of 4-year-old pit mud were divided into Cluster I (except 4B1), other locations into Cluster II, but for the 40 -year-old cellar, all locations of 
pit mud were assigned to Cluster I. There were 3 subclusters in cluster I. Subclusters (1) and (2) contained 40-year-old pit mud samples, clearly distinguishing two 40-yearold pit pools. Sub-cluster (3) mainly contained samples of 4-year-old pit mud at the under and bottom locations of the cellar. The comprehensive analysis showed that the microbial community structure of the under 4-year-old pit mud was more similar to that of the 40-year-old pit mud.

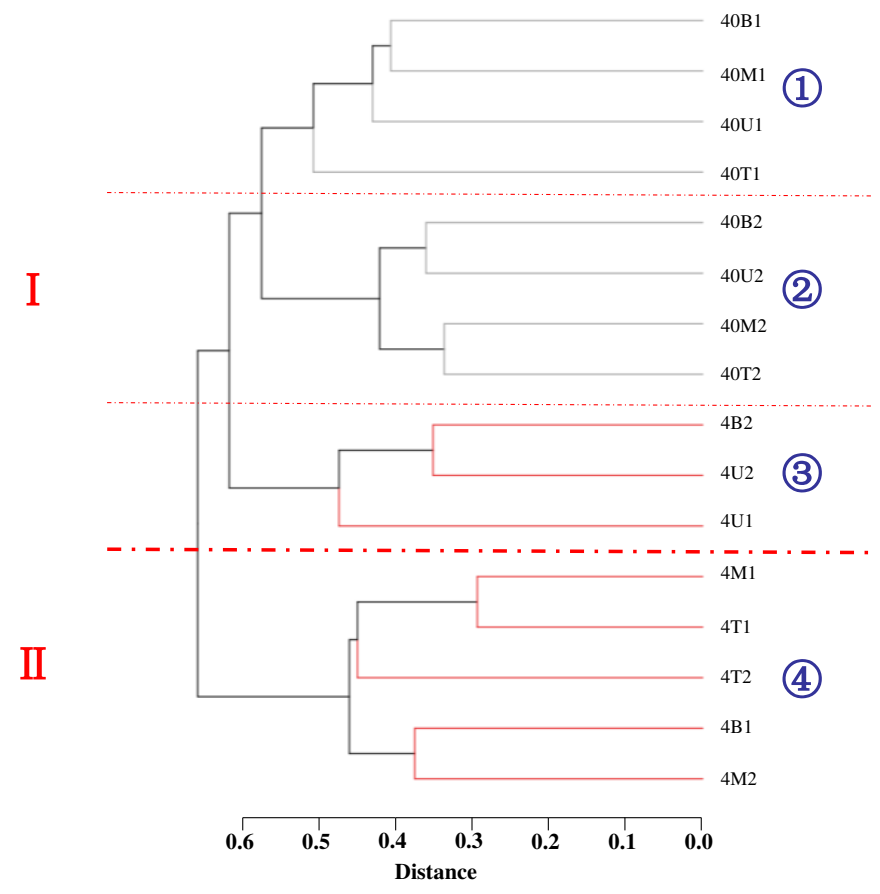

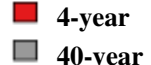

(1)

(2)

(4)

Fig 1. UniFrac distance cluster analysis based on pit-mud flora at different positions in 4-years and 40-years pits

Prokaryotic microbial community composition of different pit mud

At the phylum level, a total of 20 bacteria and 1 archaea (Euryarchaeota) were detected. More than $97 \%$ of the total abundance of samples were classified into 3 primary dominant phyla (relative abundance $\geq 1 \%$, marked with red solid lines in Fig. 2) and 6 secondarily dominant phyla(abundance $\geq 1 \%$ at least each sample, marked with a solid green line in Figure 2). The relative abundances of the dominant phyla were as follows: Firmicutes (56.70\%), Bacteroidetes (26.56\%), Synergistetes (5.28\%), 
and Euryarchaeota (3.45\%), Proteobacteria (1.81\%), Chloroflexi (1.48\%),

144

Actinobacteria (1.44\%), Cyanobacteria (1.34\%), Tenericutes (1.01\%). As against 4and 40-year-old pit mud, the microflora structure changed significantly (Fig. 2).

In the top 4-year-old pit mud, the abundances of the nine dominant phyla were all greater than $1 \%$, while in the top 40-year-old mud, Only Firmicutes dominated(67.90\%) and Actinobacteria (0.71\%), Cyanobacteria $(0.001 \%)$ and Tenericutes $(0.54 \%)$ were all less than $1 \%$.

\section{Except for the pit mud in the top part of the cellar wall, there was no significant}

difference in the microbial structure of the pit mud at the same sampling location, and the relative abundance was different. The $\mathrm{pH}, \mathrm{TA}$, and $\mathrm{NH} 4+$ content (Table 1) in the 4-year-old pit mud were significantly different from those in the 40-year-old pit mud.

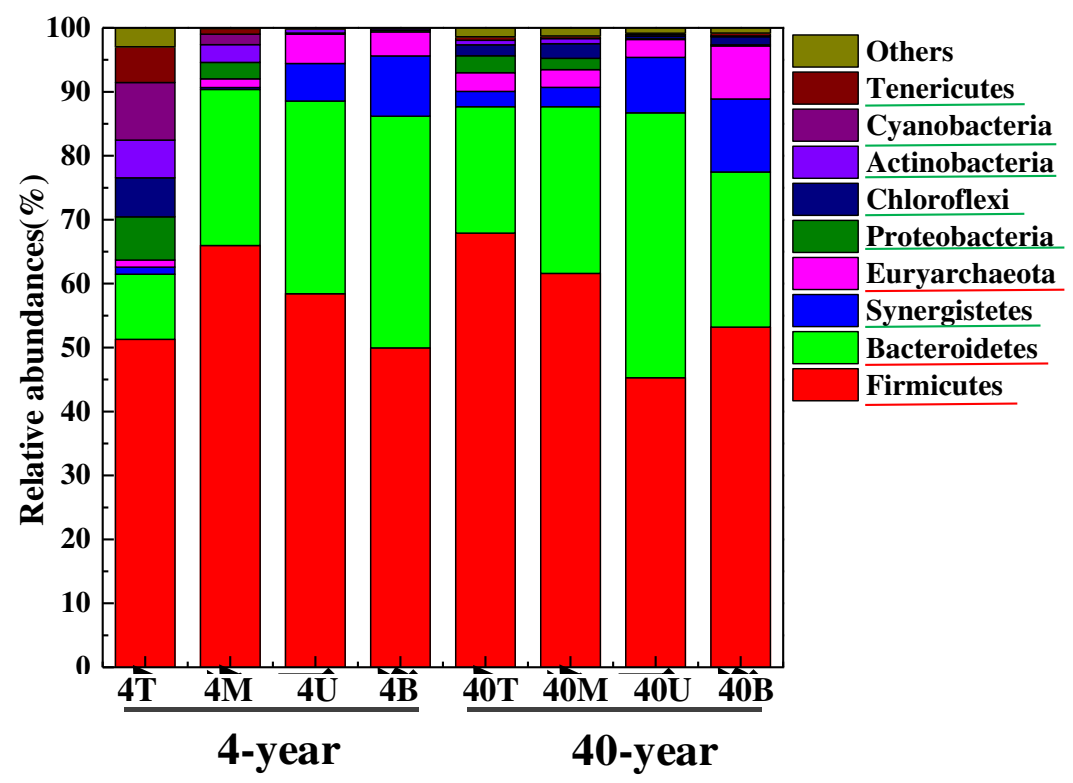

Fig 2. Composition of prokaryotic microorganisms in pit mud at the level of phylum

At the genera level, 301 genera were found. In this study, the top 10 genera (Top 10, 38 in total) of each sample were selected to reveal the diversity of microbial community composition in the pit mud from the genus classification level (Fig. 3).

The top 10 genera of each sample contained dominant genera(relative abundance> $1 \%$, 
14 with red underline). The samples were classified into three clusters: the top 4-yearold pit mud, the under 4-year-old pit mud, and the 40-year-old pit mud. Cluster (1) was a large one alone and clusters (2) and (3) were also large, which was consistent with the results in Fig. 1.

In area i (Fig. 3) in the circle, Lactobacillus was the dominant genus in 4-year-old pit mud, while the abundance was less than $1 \%$ in 40 -year-old pit mud. 8 species of bacteria including Mycoplasma, Bifidobacterium, etc. (except Lactobacillus and Bacteroides) were dominant in the top 4-year-old pit mud. However, its abundance was not detected in the 40-year-old pit mud. Bacteroides were the dominant genus only in the top 4-year-old pit mud, and Fastidiosipila and DMER64 were dominant only in 40-year-old pit mud. Both 4- and 40-year-old pit mud had unique dominant bacterial genuses. The eight genera in Christensenellaceae_R-7,Caproiciproducens, Clostridium_sensu_stricto_12, and the area ii in the circle (Fig. 3) were 4 and 40 years old. The analysis of the locations of pit mud showed us such a result that with the depth of the cellar only $36 \%$ of the dominant genuses in the 4-year-old cellar mud did not change significantly, while $79 \%$ of those in the 40 -year-old cellar mud not. As the depth of the pit deepened, The abundance of the genus of fungi in area $i$ as well as [Ruminococcus]_gauvreauii and Mesotoga genus decreased, while the abundance of the regional genus bacterium, Aminobacterium, and Methanoculleus genus in the 4year-old pit mud increased. However, with the depth of the cellar, the abundance of the 40-year-old pit mud did not change significantly. The genus Clostridium_12 always decreased. Therefore, it was inferred that its abundance was greatly affected by the locations of the pit mud. 


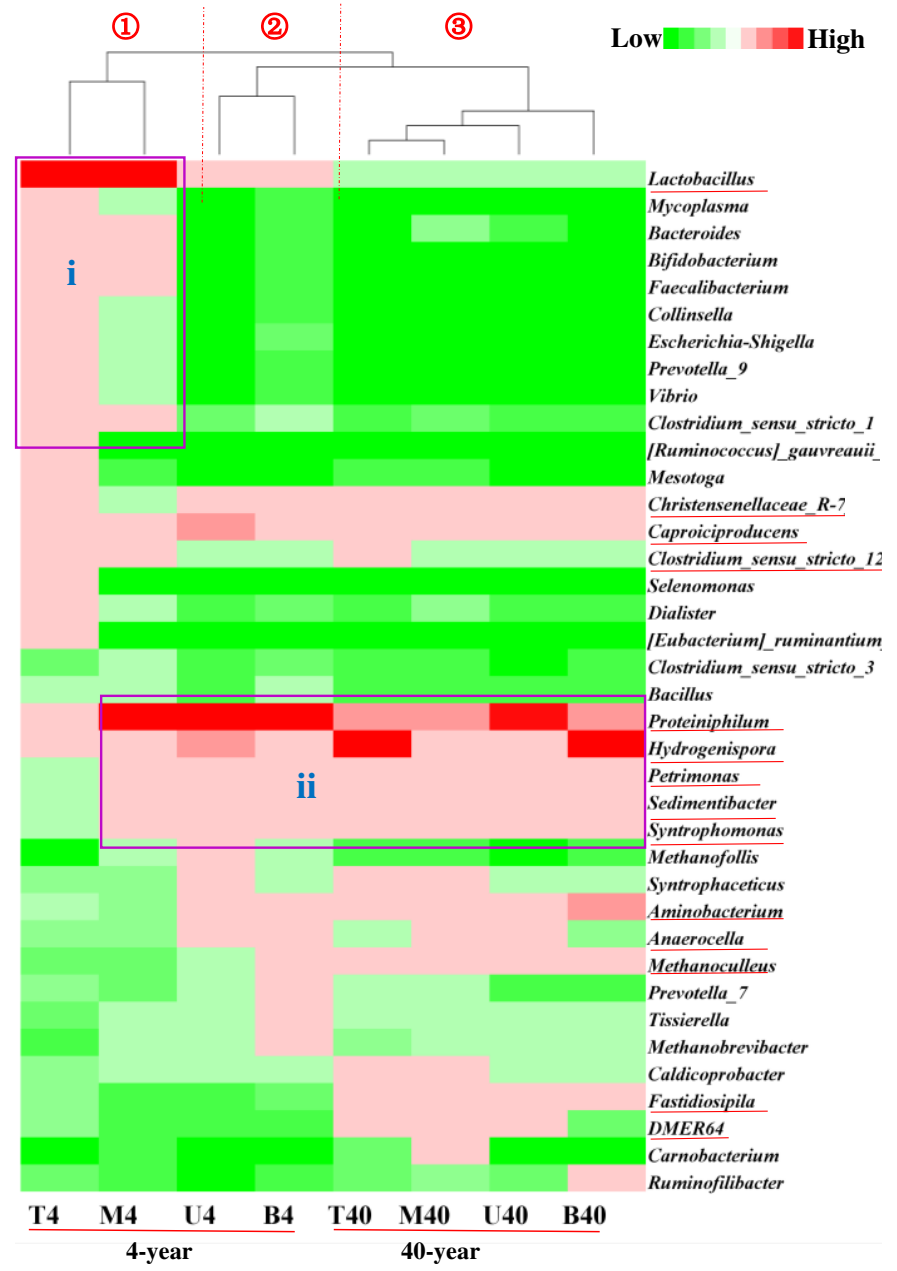

Fig 3. Heat map analysis based on Top 10 genera in each sample

The dominant bacteria of the samples (content $\geq 1 \%$ ) belonged to 11 classes. The first dominant class was Clostridia, whose dominant genera belonging to Clostridia were $46.15 \%$ in 4 -year-old pit mud, $54.39 \%$ in 40 -year-old pit mud, and $56.67 \%$ in both pit mud.

The dominant genuses (3) in different locations (Fig. 4a) of the 4-year-old pit mud outnumbered those in different locations (Fig. 4b) of the 40-year-old pit mud.

The endemic genuses were the most in the 4-year pit mud (16 species), whereas fewer in the 40-year pit mud (6 species). The physicochemical factors of the top 4-year-old pit mud were significantly different from those of other groups $(\mathrm{p}<0.05)$ (Table 1$)$, but there was no significant difference at all locations of the 40 -year-old pit mud. It implied that the differences in physicochemical factors resulted in different 

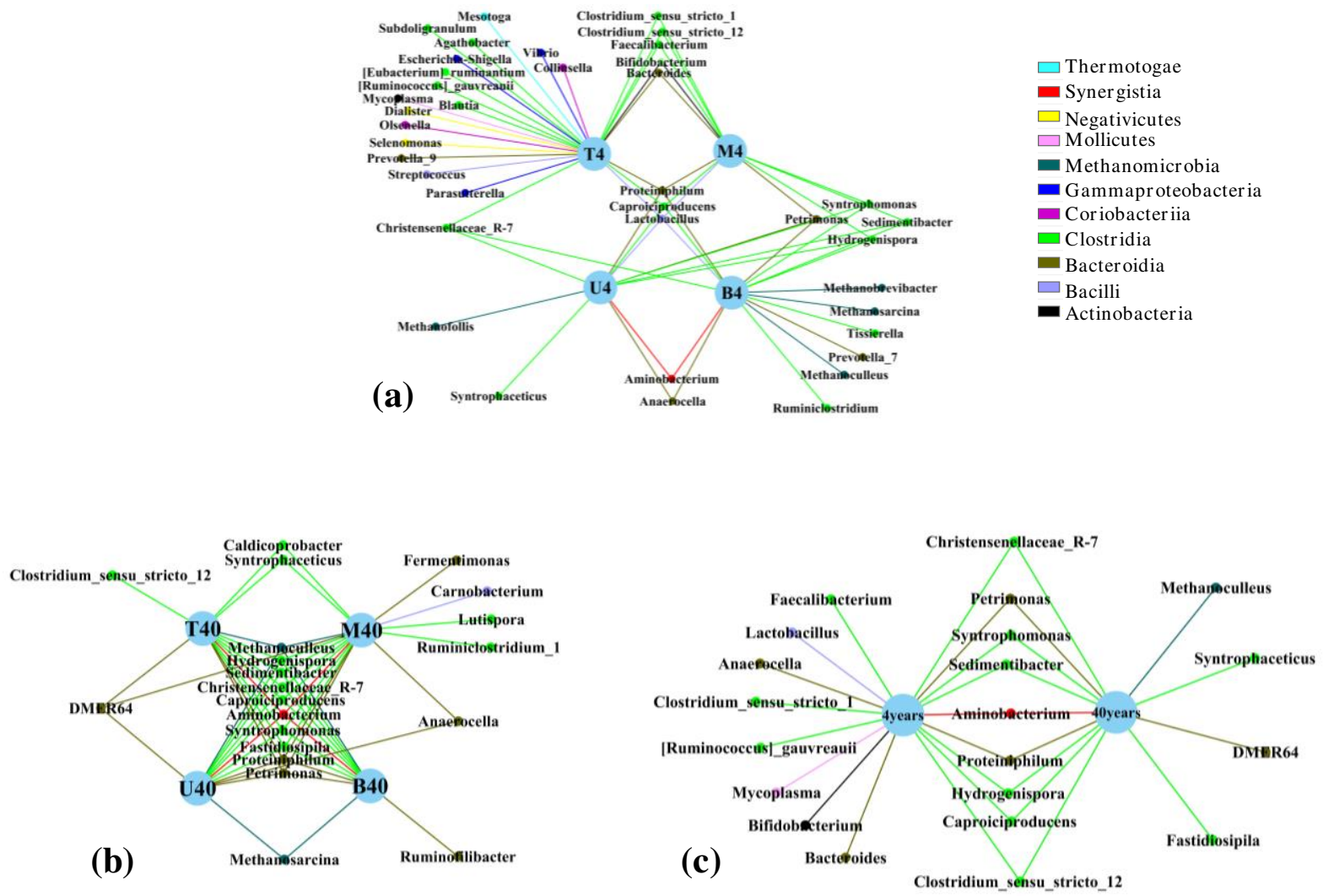

201

Fig 4. Distribution of dominant genera of different pit mud.

(a) shows the distribution characteristics of the 4-year pit mud's dominant position. (b) shows the distribution characteristics of the dominant genera of pit mud for 40-year. (c) shows the distribution characteristics of pit mud dominance in 4 and 40 years.

Network analyses of prokaryotic microbial communities in pit mud

Both positive $(\mathrm{R}>0.6$ and $\mathrm{P}<0.01)$ and negative correlations $(\mathrm{R}<-0.06$ and $\mathrm{P}<0.01)$ among prokaryotic communities, which were affiliated with 65 prokaryotic genera, were observed in this study. Based on positive correlations $(\mathrm{R}>0.6$ and $\mathrm{P}<0.01), 42$ nodes (genera) and 89 edges (pairs of significant correlations) co-occurrence patterns were detected (Fig. 5a). All nodes belonged to 12 classes, mainly concentrated in Clostridia (50\% of the total number of nodes), Bacteroides (19.05\%), Methanobacteria (7.14\%), and Gammaproteobacteria (4.76\%), and Bacillus (4.76\%). 
There were 12 hubs (nodes with more than 10 edges ) in the co-occurrence network,

which were the rare genera: Subdoligranulum, Prevotella-9, and Parasutterella,

Mycoplasma, Faecalibacterium, EscherichiaShigella, Collinsella, the 4-year pit mud hub (11.7\%).

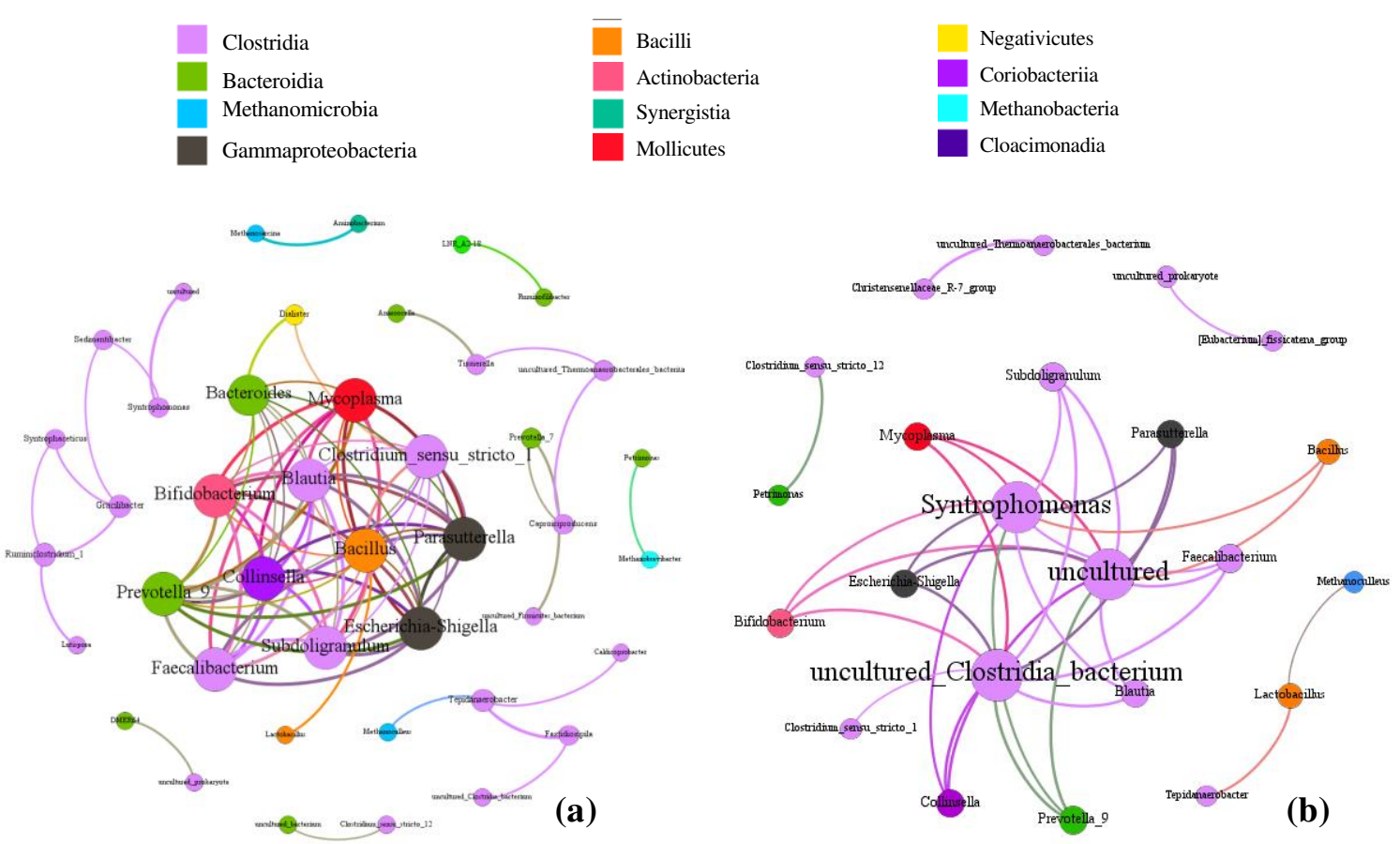

Fig 5. Networks of co-occurring prokaryotic genera in pit mud based on correlation analysis.

(a) A connection indicates a statistically significant $(\mathrm{P}<0.01)$ strongly positive correlation Spearman's $\mathrm{R}>0.8$; (b) a negative correlation Spearman's $\mathrm{R}<-0.8$. Different phyla were represented by different colors. The size of each node is proportional to the number of connections, and the thickness of each connection between two nodes is proportional to the value of Spearman's correlation coefficients of $>0.8$ or $R<-0.8$.

Based on the negative correlation network, 23 nodes and 36 edges belonged to 8 classes. Microorganisms with negative correlation in the pit mud mainly occurred in the genus Syntrophomonas, unclassified genus, and other genuses (Fig. 5b). Based on 

correlation between $\mathrm{pH}$, water content, and ammonium nitrogen $(\mathrm{P}>0.05)$.

Redundancy analysis (RDA) was performed to study the possible relationship between prokaryotic communities at the genus level and environmental variables(Fig 6). The first and second axis respectively explained $31.76 \%$ and $15.44 \%$ of the variation in prokaryotic communities and environmental factors. The samples showed the distribution and clustering of 4-year-old pit mud were more affected by its location in the cellar, whereas that of the 40-year-old pit mud was less affected. The 40-year-old pit mud from different locations of the cellar could be clustered together and its prokaryotic microbial community had obvious spatial structure characteristics, which were greatly affected by the age of the pit mud.

Conditional Term Effects was used to analyze comprehensively how each physicochemical factors contributed, which was total acid (30.9\%), AP (15.5\%), pH (6.1\%), moisture content (2.9\%), and $\mathrm{NH} 4+(2.4 \%)$, had influenced the flora structure (Fig 6a), The total acid and AP had the greatest influence on the prokaryotic microbial community and showed a significant correlation $(\mathrm{P}<0.01)$. There was no significant 


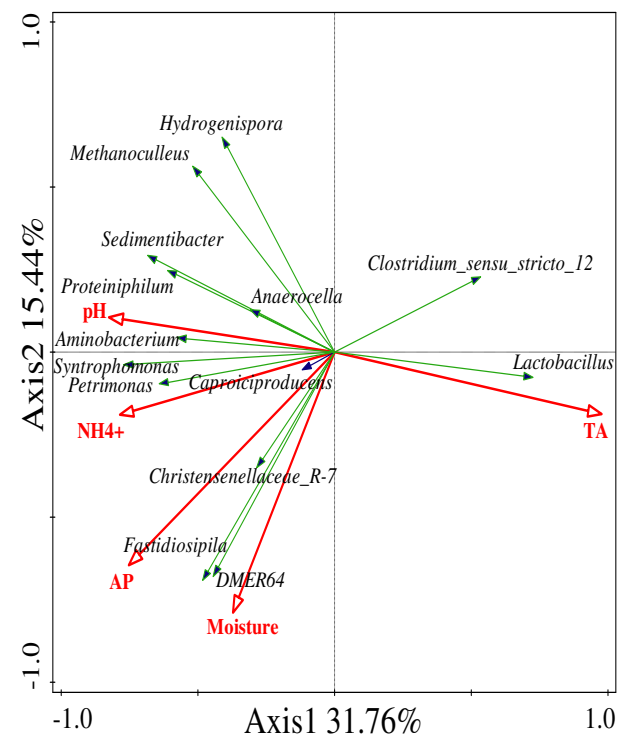

(a)

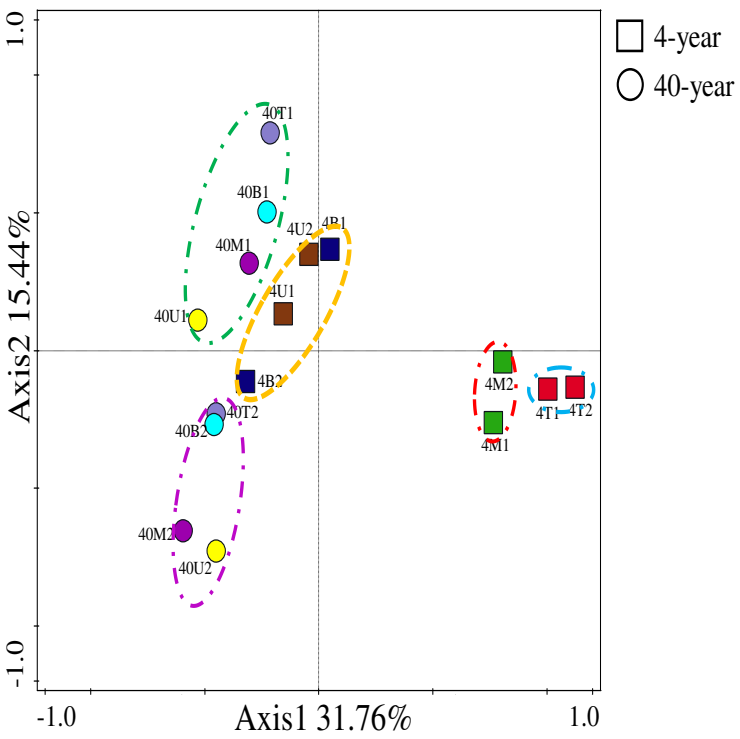

(b)

Fig 6. Redundancy analysis (RDA) of dominant microorganisms and physicochemical factors in pit mud. (a) Shows the Species and environmental variables. (b) Shows the samples.

\section{Discussion}

It is widely recognized that prokaryotic microbes in pit mud affect the quality of CSFB, and the older pit mud always produces liquor with a better taste $[5,6]$.

Previous studies have been focused on shifts of diversity and structure of prokaryotic communities; however, interactions of prokaryotic taxa in different-aged and different spatial locations of pit mud are poorly understood. Related studies have shown the $\mathrm{NH} 4+$ content of pit mud increases with its quality. $\mathrm{N}$ is an indispensable quick-acting component of microbial growth and reproduction, as it is conducive to the degradation of toxic and harmful substances from microorganisms [9]; the effective phosphorus content and the biomass of microorganisms in soil and the number of metabolites are positively correlated [10]. The $\mathrm{pH}$ value is positively correlated with the bacteria content in the pit mud $(\mathrm{p}<0.01)$ [11]. In this study (Table 1), $\mathrm{pH}, \mathrm{NH} 4+$, 
and AP in 40-year-old pit mud were higher than that in 4-year-old pit mud. It was inferred the 40-year pit mud had a higher maturity and better quality. It was consistent with the research results of Tao [6]and Zhang[8]. The alpha diversity index of the top pit mud was lower than that of the under pit mud(Table 1). On the contrary, the 40year top pit mud alpha diversity index is higher than the under pit mud. This was inconsistent with the results of Zhang and Wang et al[20,24]. Zhang et al.

[8]concluded that the diversity of pit mud on the wall of old cellars (cellar age >50 years) was lower than that at the bottom. Wang's [24] research results showed that the alpha diversity of the 6-year pit mud was higher than that of the 16-year pit mud.

Thus, it was inferred that the difference might be caused by the age and the origin of the pit mud.

Except for the pit mud on the top cellar wall, there was no significant difference in the microbial structure of the pit mud in the same sampling location, only the relative abundance was different (Fig 2). The $\mathrm{pH}$, total acid, and NH4+ content of 4-year-old pit mud on the top cellar wall were significantly different from those of 40-year-old pit mud. Physicochemical factors had an impact on the microbial community structure of pit mud $[6,8,18]$. The reason for the change in the microbial structure of the top pit mud of different aged cellars was that the physicochemical factors of the cellar mud used repeatedly for a long time had changed. The two dominant phyla at different locations of pits were Firmicutes and Bacteroidetes, which was consistent with the results of most relevant studies in China $[8,12,13,14]$. The single microbial composition in the under pit mud mainly included Firmicutes, Bacteroidetes, 
Synergistetes, and Euryarchaeota, with a relatively simple structure. The microbial diversity of pit mud on the top cellar wall was higher than that on the under cellar wall. With the depth of the pit, the abundance of Synergistetes and Euryarchaeota increased while Proteobacteria decreased.

According to the heat map in Fig 3, the relevant literature summarized $[14,15,16]$ that the abundance of Lactobacillus decreased significantly with the improvement of the quality of the pit mud and the growth of the pit age, which is the same as the results shown in this study. Hydrogenispora, Sedimentibacter, and Clostridium were the main Clostridia genus present in the pit mud [23], and their advantages were prominent in the aging and mature pit mud [15]. The correlation of pit mud quality with Sedimenlibacter and Aminobacterium was positive [18] and in high-quality pit mud [5], Methanoculleus was the dominant genus.

In summary, it was inferred, from the level of microbial community structure, that the quality of the 40-year-old pit mud and the under 4-year-old pit mud in this study was better than that of the top 4-year-old pit mud. The network analysis indicated the positive correlations of prokaryotic class, which mainly belonged to class Clostridia (Fig 5). Mycoplasma had the highest relative content in the top 4-year-old pit mud, which had been rarely reported in the past $[17,18,19,20]$. The genus Petrimonas and Syntrophomonas associated with the degraded pit mud did not appear in the hub [17]. A total of three larger independent modules and five independent modules emerged in the co-occurring network (Figure 5a). This was caused by the fact that the nodes or hubs that could connect the modules had little or no content. The connection between the hub and the micro-ecological 
stability of the pit mud was an important factor affecting the entire microbial flora network in the environment, and changes in its type and content could lead to fragmentation of the network [28]. Syntrophomonas had a negative correlation with most hubs in the positive correlation network (Fig 5b). However, the results of $\mathrm{Hu}$ et al. showed that Lactobacillus was negatively correlated with most genuses[5]. GUO [17]showed that the content of Syntrophomonas in degraded pit mud was significantly $(\mathrm{p}<0.05)$ higher than that of normal pit mud. Following this, WU[22] and others further confirmed that Syntrophomonas was detected in the third-class pit mud, but not in the first- and second-class pit mud. This indicated that the genus Syntrophomonas might accelerate the imbalance of the microbial community of the pit mud, the reduction of the robustness of the bacterial community, and the degradation of the pit mud.

RDA analysis indicated that the environmental factors, TA and AP, had significant $(\mathrm{P}<0.01)$ effects on prokaryotic communities(Fig 6). Lactic acid could cause the formation of ferrous lactate, calcium lactate, and other crystals in the pit mud, which could significantly affect the growth of the genus of bacteria in the pit mud and lead to the aging of the pit mud [20]. AP provides fast-acting P nutrients for the growth and reproduction of microorganisms in the environment and has a significant impact on the microbial community beta diversity. This further indicated that total acid and AP had a greater influence on the distribution of microbial communities in the pit mud. $85.71 \%$ of the dominant genera were distributed on the left side of the central axis of Axis1, but only two genera, Clostridium_sensu_stricto_12 and Lactobacillus, were distributed on the right side and were positively correlated with total acid (Fig. 6a). Lactobacillus was positively correlated with total acid content and negatively with $\mathrm{pH}$. 
Because Lactobacillus metabolites were mainly lactic acid, the accumulation of lactic acid could lead to an increase in total acid content and a decrease in $\mathrm{pH}$, which in turn gave rise to a deterioration in the quality of pit mud.

Methanoculleus can symbiosis with caproic acid bacteria such as Clostridium and promote the production of caproic acid by its bacteria[20]. Aminobacterium and Sedimentibacter have the function of degrading amino acids and improving ammonium nitrogen[13]. Caproiciproducens have a negative correlation with Lactobacillus. Studies have confirmed that medium- and long-chain fatty acids (such as caproic acid) produced by caproic acid bacteria can inhibit Lactobacillus [21]. In addition, Fastidiosipila mainly uses acetic acid and butyric acid as the main metabolites [25]. At present, the bacterium is rarely reported in the pit mud[26], and the genus Christensenellaceae is mainly found in the human intestine [27].

\section{Conclusions}

This study systematically compared the structures and spatial distribution characteristics of prokaryotic microbial communities between 4- and 40-year-old pit mud from Henan CSFB company. The predominant phyla of pit mud were Firmicutes, Bacteroidetes, Synergistetes, Euryarchaeota, Proteobacteria, Chloroflexi, Actinobacteria, Cyanobacteria, and Tenericutes, among which Firmicutes was the most dominant 14 genera, such as Proteiniphilum, Hydrogenispora, Lactobacillus, were principal. The proportion of endemic bacteria was higher in 4-year-old pit mud, and the number of endemic bacteria in 4-year-old pit mud on the top cellar wall was 
the highest. In 40-year-old pit mud, the dominant bacterial genuses were common in each of its locations, and the unique bacterial genuses were few. The 4-year-old pit mud on the top and under cellar wall was well distinguished, whereas the 40-year-old pit mud was well clustered. In older pits, the microbial community was less affected by the sampling locations of the pit mud. The total acid and AP $(\mathrm{P}<0.01)$ might be the main environmental factor affecting the spatial distribution of the prokaryotic microbial community of pit mud.

\section{Methods}

\section{Materials}

The pit-mud samples were taken from a well-known liquor-producing company (Jiahu

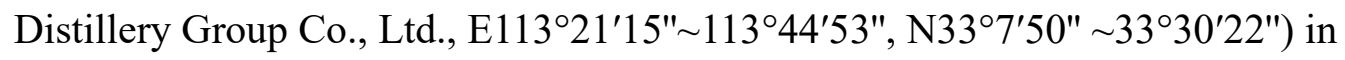
Henan Province, China. For sampling, two types of fermentation pits under normal production with different aged pit mud including young (4 years, XJ) and old (40 years, LJ) pit mud were randomly selected. Two types of pit muds were selected for each above type, and four locations varying in depth of each pit were selected, which were the top (T), middle (M), under(U), and bottom (B) cellar wall (Fig. 7). Two subsamples were collected.

Among them, 2 normal cellars (XJ 1 and XJ 2) and 2 old cellars (LJ 1) were randomly selected. LJ 2, LJ3, and LJ 4), and samples of pit mud were collected at the four locations of each cellar: the top(T), middle (M), under (U), and the bottom(B) cellar 
walls. (Figure 1). 16 representative samples (Table 2) were stored at $-30^{\circ} \mathrm{C}$ for future use.

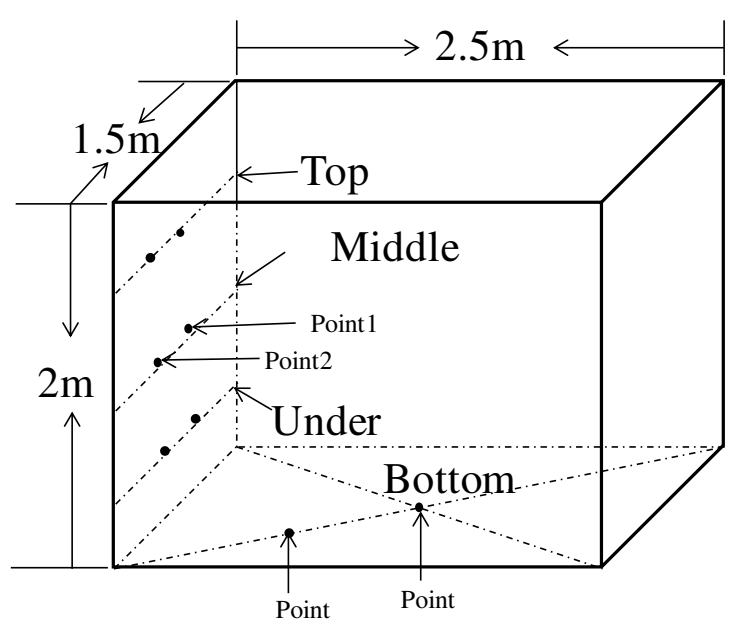

- Sampling location

Fig 7. Collection Sample Location of pit mud

Table 2. Collect samples and numbers of pit mud

\begin{tabular}{|c|c|c|c|c|}
\hline Pit mud of aged & Top & Middle & Under & Bottom \\
\hline 4-year & $4 \mathrm{~T} 1 、 \quad 4 \mathrm{~T} 2$ & $4 \mathrm{M} 1 、 \quad 4 \mathrm{M} 2$ & $4 \mathrm{U} 1 、 \quad 4 \mathrm{U} 2$ & $4 \mathrm{~B} 1 、 \quad 4 \mathrm{~B} 2$ \\
\hline 40-year & $40 \mathrm{~T} 1 、 40 \mathrm{~T} 2$ & $40 \mathrm{M} 1 、 40 \mathrm{M} 2$ & 40U1、40U2 & $40 \mathrm{~B} 1 、 40 \mathrm{~B} 2$ \\
\hline
\end{tabular}

\section{Determination of physicochemical factors}

Referring to drying methods and potentiometric methods employed by Li Junhui and others to measure the water content and $\mathrm{pH}$ value of pit mud ( $\mathrm{Li}$ et al., 2018), the acid and alkali neutralization titration method was used to determine the total acid content (Wang et al., 2012). The indigo blue colorimetric method was used to detect the content of ammonium nitrogen in pit mud (Nanjing Institute of Soil Sciences., 1987). The effective phosphorus content in pit mud was quantified referring to the NY/T1121.7-2014 standard (Agricultural Industry Standard of the People's Republic of China., 2014). Reagents included sodium hydroxide, phenolphthalein, potassium hydrogen phthalate, concentrated sulfuric acid $(\rho=1.84 \mathrm{~g} / \mathrm{mL})$, hydrochloric acid 
394

395

$(=1.19 \mathrm{~g} / \mathrm{mL})$, phenol, potassium nitroso ferricyanide, sodium perchlorate, chlorine Ammonium chloride, potassium antimony tartrate, ammonium molybdate, ammonium fluoride, boric acid (purchased from China National Pharmaceutical Group Chemical Reagent Co., Ltd.). All reagents were analytically pure. UV BlueStar A ultraviolet spectrophotometer (Leiber Tech Instruments, Beijing, China). TGL-20M high-speed refrigerated centrifuge (Lu Xiangyi Centrifuge Instrument Co., Ltd., Shanghai, China). PB-10 PH meter (sartorius, Germany). MP200A Precision Electronic Balance (Liangfeng Instrument Co., Ltd., Shanghai, China). 101-1 Electric blast drying oven (Zhongxing Weiye Instrument Co., Ltd., Beijing, China). DF-1 collector types constant temperature magnetic stirring pot (Jintan Zhongda Instrument Factory, Jiangsu, China).

\section{DNA extraction and Illumina MiSeq sequencing of 16S rRNA genes}

Magen HiPure Soil DNA Kit was used under the guidance of the step-by-step instructions to extract DNA from the pit mud samples, and the Qubit ${ }^{\circledR}$ dsDNA HS Assay Kit was used to detect the DNA concentration. The upstream primer 5'CCTACGGRRBGCASCAGKVRVGAAT-3' and downstream primer 5'GGACTACNVGGGTWTCTAATCC-3' were used to amplify the V3 and V4 regions of the 16S rRNA gene of prokaryotes. PCR amplification reaction system: TransStart Buffer $2.5 \mu \mathrm{L}$, dNTPs $2 \mu \mathrm{L}$, TransStart Taq DNA $0.5 \mu \mathrm{L}$, template $20 \mathrm{ng}$, upstream and downstream primers $1 \mu \mathrm{L}$ each, make up the volume to $25 \mu \mathrm{L}$ with ddH2O (double distilled water), reaction conditions: pre-denaturation $94^{\circ} \mathrm{C} 3 \mathrm{~min}$, 
415

416

417

418

419

420

421

422

423

424

425

426

427

Denaturation $94^{\circ} \mathrm{C} 5 \mathrm{~s}$, annealing $57^{\circ} \mathrm{C} 90 \mathrm{~s}$, extension $72^{\circ} \mathrm{C} 10 \mathrm{~s}$, final extension $72^{\circ} \mathrm{C}$ 5min, 24 cycles. Qualified PCR products were entrusted to Jinweizhi Biotechnology Co., Ltd. (Suzhou, China) for Illumina MiSeq sequencing library construction and double-end sequencing.

\section{Statistical analysis}

The Illumina MiSeq sequence was controlled using Cutadapt (v1.9.1), Vsearch (1.9.6), and Qiime (1.9.1) software. These quality controls included sequence splicing, removal of sequences containing $\mathrm{N}$, primers, and linkers, bases with a quality value $<20$ and sequences with a length of $<200 \mathrm{bp}$, and chimeras. VSEARCH (1.9.6) was used for sequence clustering for sequences that had passed quality control, where the similarity was set at $97 \%$ and classified as 1 OUT. The species taxonomic analysis on the representative sequences of OTU was performed by using the RDP classifier (Ribosomal Database Program) Bayesian algorithm, and the community composition of each sample was studied under classification levels of different species. Based on the OTU, a random leveling method for the sample sequence was used to calculate the alpha diversity indexes of the samples Shannon, Chao1, etc., the complete analysis of variance and clustering were performed using SPSS (IBM19) software. The software Origin9.0 was applied to draw a histogram of the percentage of phylum in the advantages of pit mud samples (relative abundance was more than 1\%). HemI and software Canoco 5 were used to draw Heat maps and RDA maps separately. 
437 NCBI database under BioProject PRJNA646030.

\section{Abbreviations}

439 CSFB: Chinese strong-flavor Baijiu

440 NH4+: Ammonium Nitrogen

$441 \quad$ AP: Available phosphorus

442 OTU: Operational taxonomic units

443 RDA: Redundancy analysis

\section{Authors' contributions}

445 All authors did the experiments and analyzed the data. All authors read and approved 446 the final manuscript.

\section{Acknowledgments}

448 This work was supported by the following funds: National Natural Science

449 Foundation of China (); Key Laboratory of Wuliangye-flavor liquor solid-state

450 fermentation of China National Light Industry (2018JJ017); Major Science and

451 Technology Projects in Henan Province (181100211400); and Doctoral Fund of

452 Zhengzhou University of Light Industry (2016BSJJ018).

453 Conflicts of Interest

454 The authors declare no conflicts of interest 


\section{References}

1. Yue Y Y, Zhang W X, Liu X, et al. Isolation and Identification of Facultative Anaerobes in the Pit Mud of Chinese Luzhou-flavor Liquor. Microbiology China , 2007,34(2):251-255.

2. Huang Z G, Zhen P, Luo H B. Research on Archaea Community in Luzhou-Flavor Liquor Pits. Journal of Southwest University(Natural Science Edition), 2012,32(12):91-96.

3. Liu S, Li L G, Li K, et al. Spatial Heterogeneity of Prokaryotic Microbial Communities in Luzhou-flavor Liquor Pit Mud. Food Science, 2013,34(21).

4. Li, P. Lin, W. F, Liu, X, Wang, X. W., \& Luo, L. X. Environmental factors affecting microbiota dynamics during traditional solid-state fermentation of Chinese daqu starter. Frontiers in Microbiology, 2016,7(253), 1237.

5. Hu, X. L, Du, H, Ren, C, \& Xu, Y. Illuminating anaerobic microbial community and cooccurrence patterns across a quality gradient in Chinese liquor fermentation pit muds. Applied and Environmental Microbiology, 2016,82(8), 2506-2515.

6. TAO Y, LI J, RUI J, et al. Prokaryotic communities in pit mud from different-aged cellars used for the production of Chinese strong-flavored liquor. Applied and environmental microbiology, 2014,80(7): 2254-2260.

7. Liu M K, Tang Y M, Guo X J , et al. Deep sequencing reveals high bacterial diversity and phylogenetic novelty in pit mud from Luzhou Laojiao cellars for Chinese strong-flavor Baijiu. Food Research International, 2017,102:68-76.

8. Zhang H M, Wang Y L, Meng Y J, et al. Analysis of the Bottom Pit Mud and Wall Pit Mud prokaryotic community from strong-flavor old/young mud pits. Food science, 2020,41(18):180-187.

9. Li J H, Zhang Z W, et al. Physicochemical Properties of Mud in Luzhou-flavor Liquor and Analysis of Mud Microorganism by High-throughput Sequencing. Journal of Qilu University of Technology, 2018, vol. 32, no. 06, 25-28.

10. Wang Fu Rong. Analysis and Inspection of Chinese Liquor Brewing. Beijing: Chemical Industry Press, China, 2012,27-35. 
11. Nanjing Institute of Soil Sciences, Chinese Academy of Sciences. Soil physical and chemical analysis. Shanghai: Shanghai Science and Technology Press. China, 1987.

12. Agricultural Industry Standard of the People's Republic of China. NY/T 1121.7-2014 Soil testing Part 7: Determination of available phosphorus in soil. Beijing: China Standard Press, 2014.

13. Zhang Shi Wei. Study on Microbial Community Diversity and Nitrogen Metabolism Flux of Wine Grape. Beijing: China University of Mining and Technology, 2018, 19.

14. Chen Wei. Effects of soil available phosphorus content on soil microbial biomass and metabolic activity. Changchun: Jilin Agricultural University, 2013, 22-25.

15. HE Hongkui, ZAI Hongyu, LI Anjun, et al. Research on the Relationship Between the Index of Cellar Mud and the Content of Microorganism. Liquor Making, 2019, 046(001):65-67.

16. Liu M, Deng J, Xie J, et al. Screening of Physicochemical Quality Indexes of Pit Mud Based on Correlation with Microbial Community Structure. Food Science, 2018, 039(019):44-50.

17. GUO Zhuang, GE Dong-ying, SHANG Xue-jiao, et al. Comparative Analysis on the Diversity of Bacterial Microflora in Degenerated and Normal Pit Mud. Science and Technology of Food Industry, 2018,39(22):99-104+112.

18. HU X L. Illuminating Correlation between Anaerobic Clostridial Community Diversity and Quality of Pit Mud Used for the Production of Chinese Strong-Flavor Liquor. Wuxi: Jiangnan University(JNU),2015.

19. LUO Wen, ZHANG Qianying, LIAO Zuomin, et al. Research on microbial composition of pit mud with different traits using high-throughput sequencing. Food and Fermentation Industries, 2017,043(009):9-14.

20. ZHANG Yinggang, DENG Yu. Microbial Structure and Diversity of Cellar Mud at Different Winery Cellar Age Based on High-throughput Sequencing. China Biogas, 2019(3):3-8.

21. YU Chuntao, LIU Chao, SUN Feng E, et al. Analysis of the Difference in Bacterial Communities in Pit Mud Before and After the Deterioration. Liquor-Making Science \& Technology, 2016,No.263(05):50-53. 
22. WU Shukun, LIU Mei, DENG Jie, et al. Relation of microbial composition and enzyme activity index with different grades of Luzhou flavored clay. Food and Fermentation Industries, 2018,044(001):19-24.

23. Chai LJ, Xu P X, Qian W, Zhang X J, Ma J, Lu Z M, Wang ST, Shen CH, Shi JS, Xu ZH*.. Profiling the Clostridia with butyrate-producing potential in the mud of baijiu fermentation cellar. International Journal of Food Microbiology, 2019, 297: 41-50.

24. WANG Mingyue, ZHANG Wenxue. Analysis of microbial community structure in pit mud from two Chinese Luzhou-flavor liquor producing areas. Microbiology China, 2014,041(008):1498-1506.

25. ANDERSEN S J,DE GROOF V, KHOR W C, et al. A Clostridium group IV species dominates and suppresses a mixed culture fermentation by tolerance to medium chain fatty acids products. Frontiers in bioengineering and biotechnology, 2017,5: 8 .

26. Falsen E, Collins M D, Welinder-Olsson C, et al. Fastidiosipila sanguinis gen.nov., sp nov., a new gram-positive, coccus-shaped organism from human blood. Int.J.Syst.Evol.Micr, $2005,55(2): 853-858$.

27. Jillian L. Waters, Ruth E. Ley. The human gut bacteria Christensenellaceae are widespread, heritable, and associated with health. BMC Biology, 2019,17(3).

28. FAUST K, RAES J. Microbial interactions: from networks to models. Nature Reviews Microbiology, 2012,10(8):538-550. 